\title{
Spontaneous Magnetization of Simple Metal Nanowires
}

\author{
N. Zabala,* M. J. Puska, and R. M. Nieminen \\ Laboratory of Physics, Helsinki University of Technology, P.O. Box 1100, FIN-02015 HUT, Finland
}

(Received 6 November 1997)

\begin{abstract}
On the basis of self-consistent density-functional calculations, it is predicted that three-dimensional nanowires of simple (nonmagnetic) metals undergo a transition to a spin-polarized magnetic state at critical radii. The magnetic transition also contributes to the elongation force on the nanowire. The force exhibits oscillations due to quantum confinement, in tune with the conductance steps as the wire is pulled. [S0031-9007(98)05825-6]
\end{abstract}

PACS numbers: 73.20.Dx, 71.15.Mb, 75.30.Et

A metallic nanowire is formed when two pieces of material, initially at contact, are pulled away from each other over atomic distances. In the process a connective junction elongates and narrows. In a pioneering study, Landman et al. [1] made molecular dynamics simulations for Au nanocontacts using semiempirical manyatom potentials. They showed that for wires with small radii $(R<15 \AA)$ the elongation proceeds through successive stress accumulation and relief stages. Experiments have produced nanowires via substrate-tip interactions in scanning tunnneling microscopy or in atomic force microscopy (AFM) [2]. The break-junction technique [3] is another possibility to produce nanowires. The conductance of the wire has been measured as a function of the elongation: It is found to show steplike structures, the steps being close to the conductance quantum $2 e^{2} / h$ and its integer multiples [2,3]. In AFM experiments the force needed for the elongation has been measured simultaneously with the conductance. The steps in the conductance correlate with the relief stages of the force [2].

Recently, Barnett and Landman [4] have performed first-principles molecular dynamics simulations for sodium nanowires. They showed that the neck of the pulled wire is finally only a few atoms wide and that the atomic geometries at the neck can be derived from those of isolated atomic clusters. The structure of atomic clusters of simple metals such as sodium can to a large extent be understood on the basis of the jellium model ascribing the cohesive properties to the valence electrons [5]. For finite clusters the jellium model leads to an electronic shell structure. The closed-shell configurations correspond to enhanced stability determining the clusters of "magic" atomic numbers. The jellium model has recently been applied also to nanowires [6-8]. In this Letter we report the remarkable prediction that a simple metal quantum wire, describable by the jellium model, should show spontaneous magnetization for certain wire radii. The magnetization will also be reflected in the mechanical properties of the wire.

The confinement of the conduction electrons in the direction perpendicular to the wire results in a subband structure. This is reflected as oscillations in the physical properties of the wire as its radius is varied [6-8].
The derivative of the total electronic energy with respect to wire radius gives the elongation force, and the subband structure governs the behavior of the conductance. Stafford et al. [6] applied a jellium-type model for a constriction in a cylindrical wire, and obtained with nonself-consistent electronic structure calculations a relation between the conductance and the cohesive properties. The conductance channels correspond to delocalized chemical bonds. The subband structure gives the link between the force oscillations and the conductance.

We describe a metallic nanowire within the stabilized jellium model [9]. We introduce an infinitely long cylindrical volume of rigid positive charge

$$
n+(r)=\bar{n} \theta(R-r),
$$

where $\bar{n}=3 /\left(4 \pi r_{s}^{3}\right)$ is the equilibrium bulk valence electron density, $\theta(r)$ is the Heaviside step function, and $R$ is the radius of the cylinder. The atomic units, $\hbar / m_{e} e^{2}=a_{0}$ and $m e^{4} / \hbar^{2}=1$ hartree, are used for the length and energy, respectively. The background charge is neutralized by the electron density $n_{-}(r)$ so that the electronic structure is solved self-consistently by minimizing the Kohn-Sham total energy functional of the density functional theory. We use the local spin density (LSD) approximation for the electron exchange and correlation $[10,11]$.

The effective potentials for spin up $(\uparrow)$ and spin down ( $)$ electrons are

$$
\begin{aligned}
V^{\dagger \downarrow}(r)= & \int \frac{n_{-}\left(r^{\prime}\right)-n_{+}\left(r^{\prime}\right)}{\left|r-r^{\prime}\right|} d r^{\prime}+v_{x c}^{\uparrow \downarrow}\left[n_{-}^{\uparrow}(r), n_{-}^{\downarrow}(r)\right] \\
& -\bar{n} \frac{d\left(t_{0}+\epsilon_{x c}\right)}{d \bar{n}} \theta(R-r)
\end{aligned}
$$

where the first term on the right-hand side includes the Coulomb interaction with the total electron density $n_{-}(r)=n_{-}^{\uparrow}(r)+n_{-}^{\downarrow}(r)$ and the second term gives the exchange-correlation potential within the LSD. The last term is the stabilization potential [9] containing terms due to the bulk kinetic $\left(t_{0}\right)$ and exchange-correlation $\left(\epsilon_{x c}\right)$ energies per electron. The use of the stabilization correction ensures the correct sign for the surface energy and thereby for the elongation force. 
In the infinite cylindrical geometry the electron eigenfunctions have the form

$$
\Psi_{i}^{\uparrow \downarrow}(r)=\frac{e^{i k_{z} z}}{\sqrt{L}} \frac{e^{i m \phi}}{\sqrt{2 \pi}} R_{m, n}^{\uparrow \downarrow}(r),
$$

where $k_{z}$ and $m(=0, \pm 1, \pm 2, \ldots)$ are the quantum numbers for the one-dimensional free motion in the $z$ direction and for the azimuthal $(\phi)$ dependence, respectively. $n$ is the principal quantum number for the radial wave function $R_{m, n}^{\uparrow \downarrow}(r)$ with $n-1$ nodes. $L$ is the normalization length in the $z$ direction. We solve for the radial wave functions numerically in a radial mesh. The corresponding eigenenergies are

$$
\epsilon_{i}^{\Uparrow \downarrow}=\epsilon_{|m|, n}^{\uparrow \downarrow}+\frac{k_{z}^{2}}{2} .
$$

The eigenenergies form subbands with the band bottoms at $\epsilon_{|m|, n}^{\Uparrow \downarrow}$. Different subbands are occupied up to the Fermi level $\epsilon_{F}$.

The spin densities are obtained from the eigenfunctions as

$$
n_{-}^{\Uparrow \downarrow}=\frac{1}{2 \pi^{2}} \sum_{m, n}\left|R_{m, n}^{\uparrow \downarrow}\right|^{2}\left[2\left(\epsilon_{F}-\epsilon_{|m|, n}^{\uparrow \downarrow}\right)\right]^{1 / 2} .
$$

The charge neutrality requirement, expressed in terms of the charge per unit length,

$$
\int_{0}^{\infty} n_{-}(r) 2 \pi r d r=\pi R^{2} \bar{n}
$$

defines the Fermi level. The above Kohn-Sham equations are solved fully self-consistently for the spin densities and effective potentials and the corresponding total energy of the system is determined.

We report below on calculations mimicking wires made of the Na metal, choosing the jellium density parameter $r_{s}=3.93 a_{0}$. For other $r_{s}$ values the results are similar; the spatial dependence scales approximately with the ratio $R / r_{s}$. Figure 1 shows the total electron density $n_{-}^{\uparrow}(r)+$ $n_{-}^{\downarrow}(r)$ and the spin density $n_{-}^{\uparrow}(r)-n_{-}^{\downarrow}(r)$ as a function of the distance from the cylinder axis for a jellium wire with $r_{s}=3.93 a_{0}$ (corresponding to $\mathrm{Na}$ ) and $R=5.25 a_{0}$. The ground state of this particular system is spontaneously spin polarized. The spin polarization derives from the highest occupied subband (in this case the second subband with $|m|=1$ and $n=1$ ) being fully polarized. The fact that the highest subband gives the main contribution to the spin density locates its maximum value (especially for larger $R$ values with several occupied subbands) away from the cylinder axis near the jellium edge. For the wire in Fig. 1, the spin moment is $\sim 0.3 \mu_{\mathrm{B}}$ per conduction electron (i.e., per $\mathrm{Na}$ atom). The moment per unit length is $\sim 0.1 \mu_{\mathrm{B}} / a_{0}$. The maximum moment which is found for wires with the second subband polarized is close to the value quoted above. The maximum spin moment per unit length does not vary much with $r_{s}$, but is for $2<r_{s}<$ $6 a_{0}, 0.11 \pm 0.01 \mu_{\mathrm{B}} / a_{0}$. This is due to a cancellation effect: the radius corresponding to the maximum moment increases quite linearly with $r_{s}$ decreasing the moment per unit length but the spin polarization per electron increases

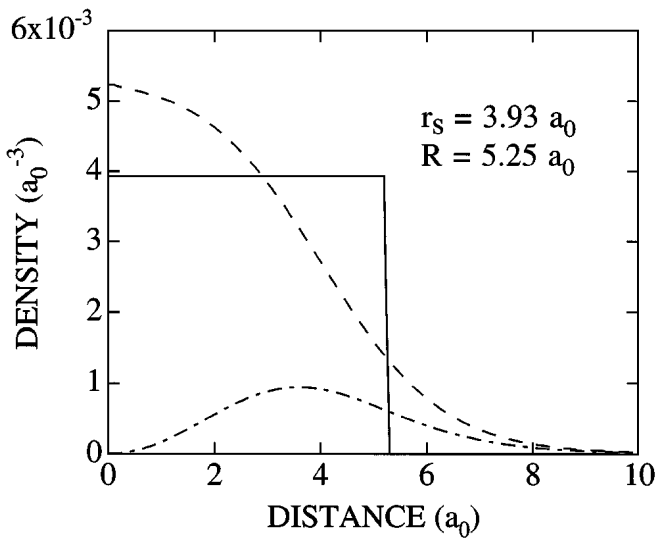

FIG. 1. Na jellium wire with $r_{s}=3.93 a_{0}$ and a radius of $5.25 a_{0}$. The total electron density $n_{-}^{\uparrow}(r)+n_{-}^{\downarrow}(r)$ (dashed line), the spin density $n_{-}^{\uparrow}(r)-n_{-}^{\downarrow}(r)$ (dash-dotted line), and the positive background charge $n_{+}$(solid line) are given as a function of the distance from the cylinder axis.

with increasing $r_{s}$. When the polarization is due to higher subbands the maximum moment per unit length decreases.

The magnetic solutions occur in the beginning of the filling of each subband. The subbands are filled in the sequence $(|m|, n)=$ $(0,1),(1,1),(2,1),(0,2),(3,1),(1,2),(4,1), \ldots, \quad$ which gives for the degeneracy ratios the sequence of $1,2,2,1,2, \ldots$. This kind of sequence has been seen in the conductance steps for Na nanowires [3]. The highest energy eigenvalues are lowered due to the total spin polarization of the highest subband. The occurrence of the magnetic moment splits also the lower energy subbands.

In order to rationalize the appearance of magnetic ground-state solutions for simple metal nanowires we proceed by studying their densities of states (DOS). For the infinite-cylinder geometry the DOS reads as

$$
\operatorname{DOS}(E)=\frac{1}{\pi R^{2}} \sum_{\substack{m, n, s \\ s=\uparrow, \downarrow}} \frac{1}{\pi \sqrt{2}} \frac{1}{\sqrt{E-\epsilon_{|m|, n}^{s}}},
$$

Thus it is a superposition of the DOS's for onedimensional subbands. The factor $\frac{1}{\pi R^{2}}$ in the front changes the DOS per unit length to the DOS per unit volume. Figure 2 shows the DOS for a $\mathrm{Na}$ wire with the radius $R=10 a_{0}$. The DOS shows a general increasing trend given by the DOS for infinite jellium. The structure with strong peaks resembles closely that of the local DOS at the neck of a nanowire modeled by a finite cluster of $\mathrm{Na}$ atoms within the pseudopotential model [4].

The magnetic solutions for jellium wires occur in the beginning of the occupancy for every new subband. Thereby the situation resembles Hund's rules for finite systems, although the number of electron states in a subband is not limited because a jellium wire is infinite in the $z$ direction. Taking the viewpoint of an infinite system it is natural to ask if the appearance of the magnetism in the jellium wires could resemble that in bulk metallic systems. The 


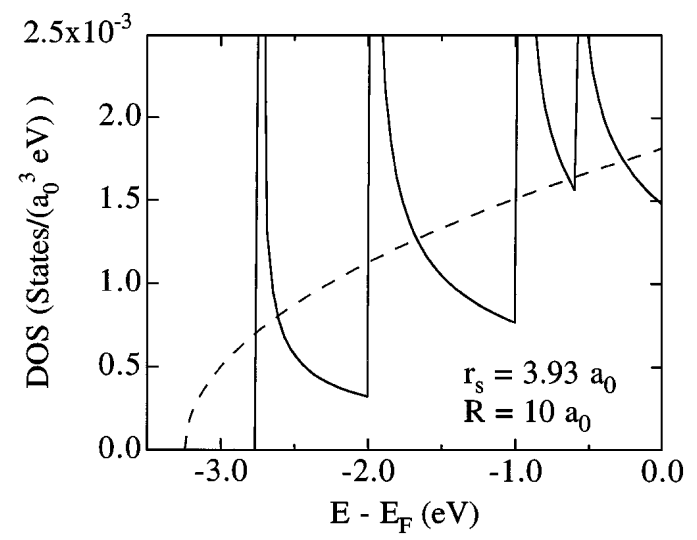

FIG. 2. Na jellium wire with $r_{s}=3.93 a_{0}$ and a radius of $10 a_{0}$. The density of states (DOS) per unit volume is given as a function of the energy. The position of the Fermi energy defines the energy zero. The DOS for the infinite jellium with $r_{s}=3.93 a_{0}$ is given for comparison (dashed line).

appearance of the ferromagnetism in metallic systems can be understood on the basis of the Stoner criterion which states that the system is ferromagnetic if

$$
I \tilde{D}\left(E_{F}\right)>1 \text {, }
$$

where $\tilde{D}\left(E_{F}\right)$ is the Fermi-level DOS per atom in a spincompensated system and $I$ is the Stoner parameter. $I$ is an "exchange" integral which includes also the electronelectron correlation effects.

The parameter $I$ should be calculated using the wave functions of the system at the Fermi level $[12,13]$. In the case of homogeneous electron gas one can define the DOS per electron and the corresponding $I$ reduces to

$$
I=\frac{8\left[\epsilon_{x c}^{F}(\bar{n})-\epsilon_{x c}^{P}(\bar{n})\right]}{9\left(2^{4 / 3}-2\right)}
$$

where $\epsilon_{x c}^{F}$ and $\epsilon_{x c}^{P}$ are the exchange-correlation energy per electron in a totally spin-polarized (ferromagnetic) and a spin-compensated (paramagnetic) electron gas, respectively. For simple metals this equation gives nearly the same results as full band calculations.

The density of states per electron, $\tilde{D}\left(E_{F}\right)$, is obtained from Eq. (7) by multiplying by the volume per electron and taking the spin degeneracy into account:

$$
\tilde{D}\left(E_{F}\right)=\frac{8 r_{s}^{3}}{3 \sqrt{2} \pi R^{2}} \sum_{m, n} \frac{1}{\sqrt{E_{F}-\epsilon_{|m|, n}}} .
$$

This equation shows that a high $r_{s}$ value (low electron density) as well as a small wire radius $R$ favor the occurrence of ferromagnetism.

The product $I \tilde{D}\left(E_{F}\right)$ is plotted in Fig. 3 as a function of the wire radius $R$. The values are contrasted to the $R$ regions (marked by black circles) where we have found stable magnetic solutions for the electronic structures. The correlation between the fulfillment of the Stoner criterion and the existence of magnetic solutions is almost quantitative. For small radii only the first subband is occupied and for the smallest radii totally spin polarized.

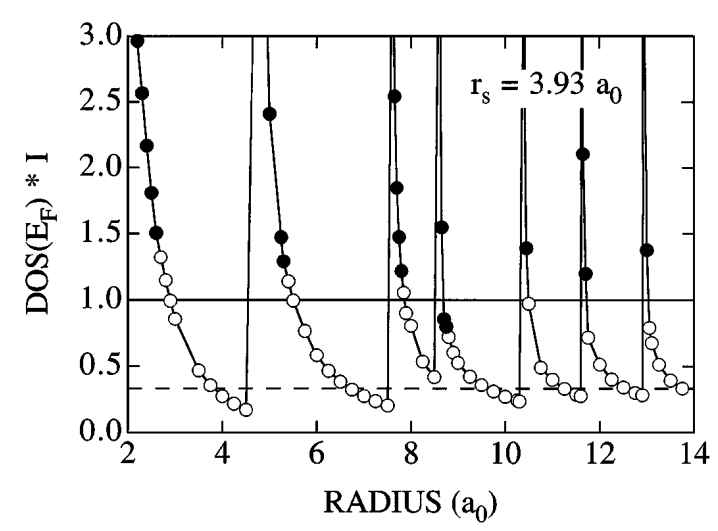

FIG. 3. Na jellium wires with $r_{s}=3.93 a_{0}$. The Stoner criterion product $I \tilde{D}$ is given as a function of the radius of the wire. The regions of stable magnetic and spin-compensated solutions are marked by black and open circles, respectively. The product for the infinite jellium with $r_{s}=3.93 a_{0}$ is given for comparison (dashed line).

When a new subband dives below the Fermi surface the Stoner criterion is fulfilled and the actual self-consistent calculations also give stable magnetic solutions. It can also be seen from the figure that the region in which the highest subband is polarized narrows in accordance with Eq. (10) when the radius $R$ increases [14].

The total energy per unit volume is shown in Fig. 4 for $\mathrm{Na}$ wires around $R=5 a_{0}$, i.e., in the region where the occupancy of the second subband is small. The calculations have been performed both by forcing the spin-up and spin-down densities to be equal (a spin-compensated local density approximation calculation) and by forcing the second subband to be fully spin-polarized and

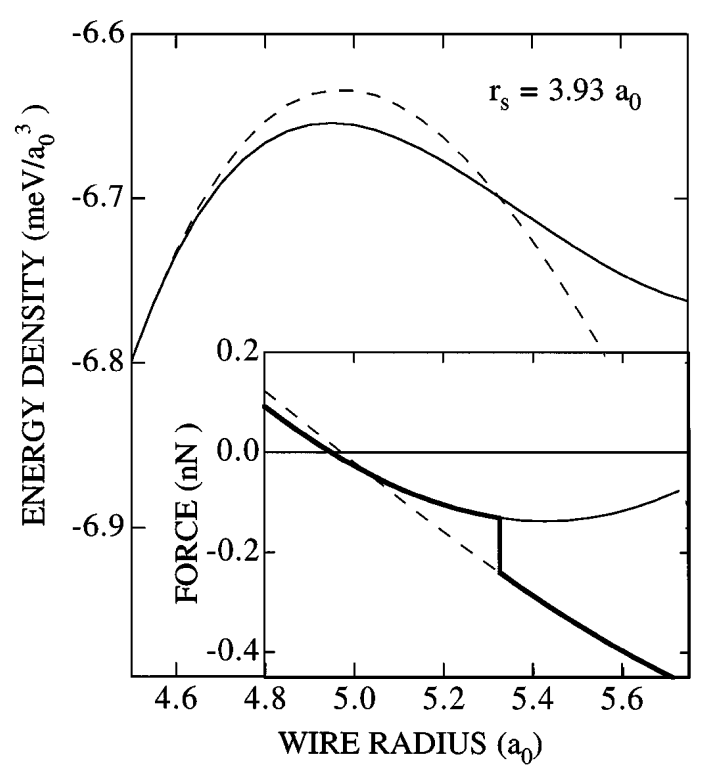

FIG. 4. Na jellium wires with $r_{s}=3.93 a_{0}$. The total energy per volume as a function of the wire radius. The second subband is occupied when the radius is larger than $\sim 4.7 a_{0}$. The energies when the second subband is fully spin polarized (solid line) and spin compensated (dashed line) are shown. The ensuing elongation forces are given in the inset. The thick line gives the adiabatic behavior of the force when the wire narrows. 
applying the LSD approximation. The latter calculation leads to lower total energies when the occupancy of the second subband is low. When the radius increases above $\sim 5.3 a_{0}$ the spin-compensated solution has a lower energy. A self-consistent calculation without any spin restrictions always finds from these two solutions the one with the lower total energy.

The elongation force or wire tension, $F=-\frac{d E_{\text {tot }}}{d L}$, is the force opposing the increase of the length of the wire at the expense of the cross sectional area. It can be calculated from the total energy per unit volume by assuming that the volume of the wire is constant during the elongation [8]. The elongation forces for spin-compensated and spinpolarized solutions are shown in the lower part of Fig. 4. When the force is negative the wire would like to shrink in length so that its radius increases. With a positive force the wire would like to spontaneously elongate and become thinner. The calculations predict that when the wire is narrowing it becomes suddenly magnetic always slightly before the emptying of the highest subband. Furthermore, this transition to the magnetic state is accompanied by a steplike decrease in the magnitude of the elongation force (a first-order transition). When the highest subband loses all its electrons the transition is smooth (a second-order transition).

The present findings have similarities with recent predictions of spin polarization in low-dimensional, quantumconfined systems. Wang and Berggren [15] considered electron states in a ribbonlike geometry mimicking quantum point contacts at the interface between two different bulk semiconductors in an external magnetic field. Because of the strong confinement perpendicular to the ribbon, the system is two dimensional. The infinite extent of the ribbon and the perpendicular confinement in the ribbon plane (parallel to the interface) leads to a subband structrure. Wang and Berggren kept the width of the ribbon constant and varied the electron density. In the case of a small external magnetic field they observed the following. At low densities only the first subband is occupied and it is first fully spin-polarized as are higher subbands occupied when the electron density increases. When the Fermi level passes the bottoms of the second and the third subband the two-dimensional electron gas of the system is fully polarized. Koskinen et al. [16] considered circular parabolic zero-dimensional quantum dots in zero external magnetic field. In this case the electron states are localized. Koskinen et al. showed that the dots with an open shell structure have ground-state spin configurations which are determined either by Hund's rules or the ground state has a zero magnetic moment but is a broken-symmetry spin-Peierls state. When just one subband is occupied the wires considered here can also undergo a spin-Peierls transition with the wave vector $q=2 k_{F}$, where $k_{F}$ is the Fermi wave vector [17]. The excitations of such a quasi-onedimensional electron gas have both a charge (plasmon) and spin-density wave branch, and related long-range correlations at $q=4 k_{F}[18]$ along the wire direction.
In summary, we have shown that the stabilized jellium model predicts the occurrence of spontaneous static magnetization in three-dimensional simple metal nanowires. The existence of the magnetism can be understood on the basis of the Stoner criterion for ferromagnetism of bulk metals. In the case of jellium wires the perpendicular confinement causes the DOS to diverge near the bottom of each subband. If the highest occupied subband is just below the Fermi level the DOS at the Fermi level can attain high values resulting in a stable spin polarization of the jellium wire. Our calculations also confirm the earlier findings [8] that the model can qualitatively account for the correlation between the conductance steps and the oscillations in the elongation force introduced by a one-dimensional subband structure. The subband structure also leads to magic radii of enhanced stability.

We are grateful to Uzi Landman, Karl-Fredrik Berggren, and Matti Manninen for useful communications. N.Z. acknowledges support from the Spanish Ministry of Education (DGES), Project No. PR95-591, and the Laboratory of Physics of the HUT for the hospitality.

*Permanent address: Elektrika eta Elektronika Saila, Zientzi Fakultatea, E.H.U-U.P.V 644 P.K., 48080 Bilbo, Spain.

[1] U. Landman, W. D. Luedtke, N. Burnham, and R. J. Colton, Science 248, 454 (1990).

[2] G. Rubio, N. Agrait, and S. Vieira, Phys. Rev. Lett. 76, 2302 (1996).

[3] J. M. Krans et al., Nature (London) 375, 767 (1995).

[4] R. N. Barnett and U. Landman, Nature (London) 387, 788 (1997).

[5] W. A. de Heer, Rev. Mod. Phys. 65, 611 (1993); M. Brack, ibid. 65, 677 (1993).

[6] C. A. Stafford, D. Baeriswyl, and J. Bürki, Phys. Rev. Lett. 79, 2863 (1997).

[7] J. M. van Ruitenbeek, M.H. Devoret, D. Esteve, and C. Urbina, Phys. Rev. B 56, 12566 (1997).

[8] C. Yannouleas and U. Landman, J. Phys. Chem. B 101, 5780 (1997).

[9] J. P. Perdew, H. Q. Tran, and E. D. Smith, Phys. Rev. B 42, 11627 (1990).

[10] D. M. Ceperley and B. J. Alder, Phys. Rev. Lett. 45, 566 (1980).

[11] J. Perdew and A. Zunger, Phys. Rev. B 23, 5048 (1981).

[12] J. F. Janak, Phys. Rev. B 16, 255 (1977).

[13] O. Gunnarsson, J. Phys. F 6, 587 (1976).

[14] For wires with finite length $(L)$, the DOS singularities become rounded, in a way dependent on the termination. However, the analysis presented here is valid as long as $k_{F} / L$ is small compared to the subband spacing. For wires with two or three subbands, this means $L>2 r_{s}$.

[15] C.-K. Wang and K.-F. Berggren, Phys. Rev. B 54, R14 257 (1996).

[16] M. Koskinen, M. Manninen, and S. M. Reimann, Phys. Rev. Lett. 79, 1389 (1997).

[17] M. Manninen (private communication).

[18] H. J. Schulz, Phys. Rev. Lett. 71, 1864 (1993). 